\title{
Tests de détection rapides d'antigènes pour le SRAS-CoV-2
}

\author{
Michael Liu BA, Rahul K. Arora BScS, Mel Krajden MD
}

Citation : CMAJ 2021 March 29;193:E447. doi : 10.1503/cmaj.202827-f; diffusion hâtive le 3 mars 2021

Voir la version anglaise de l'article ici : www.cmaj.ca/lookup/doi/10.1503/cmaj.202827; voir l'analyse connexe en anglais ici : www.cmaj.ca/lookup/doi/10.1503/cmaj.210100, et en français ici : www.cmaj.ca/lookup/doi/10.1503/cmaj.210100-f

1 Les tests de détection rapides d'antigènes (TDRA) pour le coronavirus du syndrome respiratoire aigu sévère 2 (SRAS-CoV-2) sont des immunodosages cliniques modérément sensibles et hautement spécifiques

Les TDRA détectent les antigènes du SRAS-CoV-2 dans des échantillons prélevés des voies respiratoires supérieures et fournissent le résultat dans un intervalle de 15-30 minutes. L'éventail de sensibilité de ces tests de détection est de $30 \%-80 \%$ et leur gamme de spécificité est de $98 \%-100 \%$, comparativement au test d'amplification en chaîne par polymérase couplé à une transcription inverse (RT-PCR) ${ }^{1}$.

\section{Les TDRA peuvent cibler de façon adéquate les} personnes contagieuses

Les TDRA peuvent détecter l'infection chez les personnes porteuses d'une charge virale associée à la transmission du SRAS-CoV-2, qu'elles soient symptomatiques ou non ${ }^{2}$. Par exemple, le TDRA Panbio présente une sensibilité d'environ $97 \%$ chez les personnes porteuses d'une charge virale plus élevée (le cycle seuil du test d'amplification RT-PCR nécessaire pour détecter l'ARN étant $<25$ ) et d'environ $42 \%$ chez celles dont la charge virale est faible (cycle seuil $>25)^{3}$.

\section{Les TDRA sont utiles dans les programmes de dépistage afin de briser la chaîne de transmission}

Les personnes atteintes de la forme asymptomatique ou présymptomatique de l'infection peuvent représenter plus de la moitié des cas de transmission du SRAS-CoV-2, mais souvent elles ne sont pas admissibles à un test diagnostique à moins d'être entrées en contact étroit avec un cas confirmé ${ }^{4}$. Les programmes de dépistage qui emploient les TDRA ciblent les personnes qui sont asymptomatiques afin de repérer rapidement celles qui sont porteuses d'une charge virale élevée, ce qui est tout particulièrement important pour briser la chaîne de transmission dans les milieux à haut risque et les milieux de vie collectifs ${ }^{5,6}$.

\section{La probabilité prétest devrait guider \\ l'interprétation des résultats des TDRA}

Pour les personnes présentant une plus grande probabilité prétest d'infection au SRAS-CoV-2 (p. ex., les personnes qui sont symptomatiques, celles qui ont récemment été exposées au virus ou celles vivant dans un milieu où la prévalence de l'infection est élevée), les résultats positifs de TDRA devraient être pris en charge comme des cas confirmés. Les résultats pourraient être faussement négatifs chez les personnes présentant une faible charge virale et devraient être confirmés par un test RT-PCR. Pour les personnes dont la probabilité prétest est faible, les résultats positifs aux TDRA devraient tout d'abord être pris en charge comme des cas confirmés, avec une vérification des résultats par RT-PCR afin d'éliminer les résultats faussement positifs. Les résultats négatifs obtenus dans ce groupe peuvent signifier que l'infection est inexistante ou qu'une infection avec une faible charge virale est présente, donc les personnes devraient continuer de suivre les mesures usuelles de la santé publique afin d'éviter de contracter et de transmettre l'infection.

\section{5}

La formation des administrateurs de tests de dépistage en lien avec la collecte d'échantillons et les procédures d'analyse améliore l'exactitude et la sécurité des tests.

Une formation adéquate en collecte d'échantillons améliore la sensibilité et l'exactitude des tests de dépistage ${ }^{6}$. Les membres du personnel doivent aussi être formés à l'utilisation de l'équipement de protection individuelle et aux procédures de sécurité biologique appropriées afin de prévenir l'infection lors du prélèvement et de la manipulation des échantillons.

\section{Références}

1. Dinnes J, Deeks JJ, Adriano A, et al. Rapid, point-of-care antigen and molecularbased tests for diagnosis of SRAS-CoV-2 infection. Cochrane Database Syst Rev 2020;(8):CD013705. 
2. Marks M, Millat-Martinez P, Ouchi D, et al. Transmission of COVID-19 in 282 clusters in Catalonia, Spain: a cohort study. Lancet Infect Dis 2021; S1473-3099:30985-3.

3. Linares M, Pérez-Tanoira R, Carrero A, et al. Panbio antigen rapid test is reliable to diagnose SRAS-CoV-2 infection in the first 7 days after the onset of symptoms. J Clin Virol 2020;133:104659.

4. Subramanian R, He Q, Pascual M. Quantifying asymptomatic infection and transmission of COVID-19 in New York City using observed cases, serology, and testing capacity. Proc Natl Acad Sci U S A 2021;118:e2019716118.

5. Priority strategies to optimize testing and screening for COVID-19 in Canada: Report. Ottawa: Government of Canada; 2021.

6. Crozier A, Rajan S, Buchan I, et al. Put to the test: use of rapid testing technologies for COVID-19. BMJ 2021;372:n 208.

Intérêts concurrents : Mel Krajden a reçu des subventions versées à son établissement par Roche, Hologica et Siemens, indépendamment des travaux soumis. Aucun autre intérêt concurrent n'a été déclaré.

Cet article a été révisé par des pairs.

Affiliations : Faculté de médecine de Harvard (Liu), Boston, Mass.; Unity Health Toronto (Liu), Hôpital St. Michael, Toronto, Ont.; Institut de génie biomédical (Arora), Université d'Oxford, Oxford, R.-U.; Département des sciences en santé communautaire (Arora), Université de Calgary, Calgary, Alb.; Département de pathologie et médecine de laboratoire (Krajden), Université de la ColombieBritannique; Centre de contrôle des maladies de la ColombieBritannique (Krajden), Vancouver, C.-B.

Propriété intellectuelle du contenu : Il s'agit d'un article en libre accès distribué conformément aux modalités de la licence Creative Commons Attribution (CC BY-NC-ND 4.0), qui permet l'utilisation, la diffusion et la reproduction de tout médium à la condition que la publication originale soit adéquatement citée, que l'utilisation se fasse à des fins non commerciales (c.-à-d., recherche ou éducation) et qu'aucune modification ni adaptation n'y soit apportée. Voir : https://creativecommons.org/licenses/by-nc-nd/4.0/deed.fr.

Remerciements : Les auteurs remercient Irfan Dhalla pour ses observations éclairées sur des versions antérieures de cet article.

Correspondance : Mel Krajden, Mel.Krajden@bccdc.ca 